\title{
Early Detection of Ovarian Cancer using the Risk of Ovarian Cancer Algorithm with Frequent CA125 Testing in Women at Increased Familial Risk - Combined Results from Two Screening Trials
}

Steven J. Skates*1, Mark H. Greene ${ }^{\star 2}$, Saundra S. Buys ${ }^{3}$, Phuong L. Mai², Powel Brown ${ }^{4}$, Marion Piedmonte $^{5}$, Gustavo Rodriguez ${ }^{6}$, John O. Schorge ${ }^{1}$, Mark Sherman², Mary B. Daly ${ }^{7}$, Thomas Rutherford $^{8}$, Wendy R. Brewster ${ }^{9}$, David M. O'Malley ${ }^{10}$, Edward Partridge ${ }^{11}$, John Boggess ${ }^{12}$, Charles W. Drescher ${ }^{13}$, Claudine Isaacs ${ }^{14}$, Andrew Berchuck ${ }^{15}$, Susan Domchek ${ }^{16}$, Susan A. Davidson ${ }^{17}$, Robert Edwards ${ }^{18}$, Steven A. Elg ${ }^{19}$, Katie Wakeley ${ }^{20}$, Kelly-Anne Phillips ${ }^{21,22,23}$, Deborah Armstrong ${ }^{24}$, Ira Horowitz ${ }^{25}$, Carol J. Fabian ${ }^{26}$, Joan Walker ${ }^{27}$, Patrick M. Sluss ${ }^{1}$, William Welch ${ }^{28}$, Lori Minasian², Nora K. Horick ${ }^{1}$, Carol H. Kasten ${ }^{29}$, Susan Nayfield ${ }^{30}$, David Alberts ${ }^{31}$, Dianne M. Finkelstein ${ }^{+1}$, Karen $\mathrm{H}$. Lut4. ${ }^{\star}$ Contributed equally, ${ }^{\dagger}$ contributed equally.

${ }^{1}$ Massachusetts General Hospital, Boston, Massachusetts. ${ }^{2}$ National Cancer Institute, Rockville, Maryland. ${ }^{3}$ Huntsman Cancer Institute, University of Utah School of Medicine, Salt Lake City, Utah. ${ }^{4} \mathrm{MD}$ Anderson Cancer Center, Houston, Texas. ${ }^{5}$ Roswell Park Cancer Institute, Buffalo, New York. ${ }^{6}$ NorthShore University Health System, Evanston, IL. ${ }^{7}$ Fox Chase Cancer Center, Philadelphia, Pennsylvania. ${ }^{8}$ Western CT Health Network, Danbury, Connecticut. ${ }^{9}$ University of North Carolina, Chapel Hill, Chapel Hill, North Carolina. ${ }^{10}$ Ohio State University and the James Cancer Center, Columbus, Ohio. ${ }^{11}$ University of Alabama at Birmingham, Comprehensive Cancer Center, Birmingham, Alabama. ${ }^{12}$ Rex Cancer Center, Raleigh, North Carolina. ${ }^{13}$ Fred Hutchinson Cancer Research Center, Seattle, Washington. ${ }^{14}$ Georgetown University Medical Center, Lombardi Cancer Center, Washington DC. ${ }^{15}$ Duke University Medical Center, Division of Gynecologic Oncology, Durham, North Carolina. ${ }^{16}$ University of Pennsylvania, Abramson Cancer Center, Philadelphia, Pennsylvania. ${ }^{17}$ Denver Health Medical Center, Denver, Colorado. ${ }^{18}$ Magee-Womens Hospital, Pittsburgh, Pennsylvania. ${ }^{19}$ The lowa Clinic, Gynecologic Oncology, Des Moines, lowa. ${ }^{20}$ DanaFarber Cancer Center in Clinical Affiliation with South Shore Hospital, South Weymouth, Massachusetts. ${ }^{21}$ Division of Cancer Medicine, Peter MacCallum Cancer Centre, Melbourne, Australia, ${ }^{22}$ Sir Peter MacCallum Department of Oncology, The University of Melbourne, Melbourne, Australia, ${ }^{23}$ Centre for Epidemiology and Biostatistics, Melbourne School of Population and Global Health, The University of Melbourne, Melbourne, Australia, ${ }^{24}$ Johns Hopkins Kimmel Cancer Center, Baltimore, Maryland. ${ }^{25}$ Emory University School of Medicine, Atlanta, Georgia. ${ }^{26}$ The University of Kansas Cancer Center, Westwood, Kansas. ${ }^{27}$ Stephenson Cancer Center, University of Oklahoma HSC, Oklahoma City, Oklahoma. ${ }^{28}$ Brigham and Women's Hospital, Boston, Massachusetts. ${ }^{29} \mathrm{Food}$ and Drug Administration, Silver Spring, Maryland. ${ }^{30}$ University of Florida, Gainesville, Florida. ${ }^{31}$ University of Arizona Cancer Center, Tucson, Arizona.

Running Title: Ovarian cancer early detection: ROCA in increased risk women

Keywords: Early detection, cancer screening, ovarian cancer, biomarker algorithm, BRCA1/2 Financial Support: The ROCA study was supported mainly by research grants/contracts from NCI to sites in the Cancer Genetics Network, the Ovarian SPORE program, and the Early Detection Research Network (CA078284 D. Finkelstein, CA078134 H. Anton-Culver, CA078164 D. Bowen, CA078156 S. Domchek, CA078148 C. Griffin, CA078146 C. Isaacs, CA078174 G. Mineau, CA078157 J. Schildkraut, CA078142 L. Strong, HHSN2612007440000C D. Finkelstein, CA083638 R. Ozols, CA083591 E. Partridge, CA086389 H. Lynch); Fujirebio Diagnostics Inc supported the CGN study for one year after NCl funding ended. Drs. P. Mai and M. Greene were supported by the Intramural Research Program, NCI/NIH. The Gynecologic Oncology Group's study (GOG-0199) was supported by intramural research funds from the Clinical Genetics Branch, and National Cancer 
Institute grants to the Gynecologic Oncology Group (GOG) Administrative Office and Tissue Bank (CA027469 P. Di Saia), the GOG Statistical and Data Center (CA037517 J. Blessing), and by NCl's Community Clinical Oncology Program (CCOP) grant (CA101165 P. Di Saia). Participation by the investigators of the Australia and New Zealand Gynaecological Oncology Group (ANZGOG) is gratefully acknowledged. K-A. Phillips is an Australian National Breast Cancer Foundation Fellow. Corresponding Author: Steven Skates, 50 Staniford Street, Suite 560, Massachusetts General Hospital, Boston MA 02114. sskates@partners.org.

Conflicts of Interest: Steven Skates is a co-developer of the risk of ovarian cancer algorithm. Massachusetts General Hospital has licensed software implementing the algorithm.

Word Count: Text= 5,084; Abstract $=249$; Figures, Tables: 3; Statement of Translational Relevance $=149$ 


\section{Statement of Translational Relevance}

With further evidence from larger cohorts, the risk of ovarian cancer algorithm (ROCA) based on longitudinal serum CA125 measurements could be applied to women at increased genetic/familial risk who elect screening for early detection of ovarian cancer. ROCA personalizes screening to a woman's unique CA125 level, using each woman as her own control, resulting in a more precise screening test. Shifting transvaginal ultrasound from concurrent with CA125 (the present standard) to secondary triage only in women with abnormal ROCA results greatly reduces ultrasound-related false-positive tests. However, consideration of risk-reducing salpingo-oophorectomy (RRSO) upon completion of child bearing and when ovarian cancer risk increases above population risk is and should remain the current standard of care for $B R C A 1 / 2$ mutation carriers. If further studies determine ROCA is effective, this improved approach to early detection would be available to increased risk women who choose, despite a strong recommendation for RRSO, to postpone their surgery. 


\section{Abstract}

Purpose: Women at familial/genetic ovarian cancer risk often undergo screening despite unproven efficacy. Research suggests each woman has her own CA125 baseline; significant increases above this level may identify cancers earlier than standard 6-12 monthly CA125>35U/mL.

Experimental Design: Data from prospective Cancer Genetics Network and Gynecologic Oncology Group trials, which screened 3,692 women (13,080 woman-screening years) with a strong breast/ovarian cancer family history or BRCA1/2 mutations, were combined to assess a novel screening strategy. Specifically, serum CA125 q3 months, evaluated using a risk of ovarian cancer algorithm (ROCA), detected significant increases above each subject's baseline, which triggered

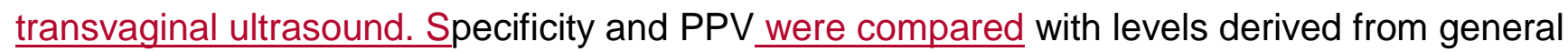
population screening (specificity 90\%, PPV 10\%), and stage-at-detection was compared with historical high-risk controls.

Results: Specificity for ultrasound referral was $92 \%$ vs. 90\% ( $\mathrm{p}=0.0001)$, and PPV was $4.6 \%$ vs. $10 \%$ $(p>0.10)$. Eighteen of 19 malignant ovarian neoplasms (prevalent $=4$, incident $=6, R R S O=9$ ) were detected via screening or risk-reducing salpingo-oophorectomy (RRSO). Amongst incident cases (which best reflect long-term screening performance), 3/6 invasive cancers were early-stage (I/II) (50\% versus $10 \%$ historical $B R C A 1$ controls; $p=0.016)$. Six of 9 RRSO-related cases were stage I. ROCA flagged $3 / 6(50 \%)$ incident cases before CA125 exceeded $35 \mathrm{U} / \mathrm{mL}$. Eight of 9 stages $0 / 1 / I I$ ovarian cancer patients were alive at last follow-up (median 6 years).

Conclusions: For screened women at familial/genetic ovarian cancer risk, ROCA q3 months had better early-stage sensitivity at high specificity, and low yet possibly acceptable PPV compared with CA125>35 U/mL q6/q12 months, warranting further larger cohort evaluation. 


\section{Introduction}

Early detection of ovarian cancer with periodic CA125 blood tests and transvaginal ultrasound (TVU) was recommended in the US, $(1,2)$ but not universally,(3) for women at increased familial risk. Recently, the US recommendation evolved to a consideration(4) including investigational screening studies(5) since standard use of CA125 and TVU showed no screening efficacy(6-12), with most cases detected in late-stage disease. $(13,14)$ We conducted two pilot detection trials in women at increased familial risk to test a new approach to screening, estimating specificity, positive predictive value (PPV), and comparing the proportion detected in early-stage with the proportion in high-risk historical controls. To increase the proportion detected in early-stage while maintaining high test specificity, the new approach (i) personalized screening by detecting significant rises above each woman's CA125 baseline, (ii) tested CA125 more frequently, and (iii) referred women to TVU only following a positive blood test.

Previous CA125-based screening studies in women at familial risk have shown no improvement in outcomes, perhaps because they: (i) classified tests as positive only when CA125 >35 U/mL; (ii) tested annually; and (iii) tested with concurrent CA125 and TVU, which creates high false-positive rates from frequent TVUs. We addressed the first limitation by leveraging longitudinal CA125 data based on studies indicating that each woman's baseline CA125 level is unique.(15-17) ROCA identifies significant rises above each individual's baseline, personalizing the test and increasing the likelihood of earlier disease detection, ideally before reaching the $35 \mathrm{U} / \mathrm{mL}$ threshold.(16) Specificity is maintained by ruling out women with high, stable CA125 values. Further exemplifying the need for a personalized approach we note there are significant differences between the CA125 distributions for post-menopausal $\left(98^{\text {th }}\right.$ percentile $=35 \mathrm{U} / \mathrm{mL}$ ) and pre-menopausal (98th percentile $=52 \mathrm{U} / \mathrm{mL}$ ) women.(18) 
Regarding the second limitation, most previous screening studies of increased-risk women evaluated annual testing schedules. Data regarding more frequent testing are limited.(19) Ovarian cancer progression from early- to late-stage disease may be too rapid for annual testing to effectively detect early-stage disease.(20) More frequent blood tests might increase the likelihood of detecting earlystage disease.

Finally, simultaneous testing with CA125 and TVU yields high false-positive rates, primarily due to benign TVU-detected adnexal masses, necessitating many surgeries to identify one true-positive.(21) Preliminary data suggest that using TVU as a secondary test to evaluate abnormal CA125 tests might significantly reduce false-positive rates.(22-26) Thus, our studies included annual TVU for all subjects (standard care during conduct of studies), and interim TVU only to assess abnormal ROCA results.

The two prospective pilot early detection studies reported here screened increased-risk women using CA125-based ROCA every three months, interval TVU only for abnormal ROCA results, and annual screening TVU, per standard care. We use the term "increased-risk" versus "high-risk" advisedly, to more accurately reflect participant risk heterogeneity, with a high-risk subset (BRCA1/2 mutation carriers) and groups at intermediate risk between mutation carriers and the general population (unknown mutation status or mutation-negative/strong family-history-positive subjects). The CGN and GOG studies implemented the same screening protocol and eligibility criteria; US and Australian CA125 testing was done in one centralized research laboratory in each country. Multiple duplicate test samples were analyzed to ensure high inter-lab concordance. Study goals included evaluating compliance with the quarterly CA125 screening schedule and obtaining estimates of ROCA performance characteristics through a pre-planned combined analysis of the two studies. 
Performance characteristics listed for the CGN study were sensitivity, specificity, and PPV, for the GOG study were specificity and PPV, while the combined analysis specified goals of achieving a specificity of at least $90 \%$ and a PPV of at least $10 \%$ (see Supplement). Optimal debulking was added as a study endpoint after study initiation.

\section{Methods}

These two studies have been described previously. $(18,27)$ The primary outcomes were specificity, PPV, and sensitivity for invasive ovarian cancer.

\section{Subjects}

The Cancer Genetics Network (CGN) initiated the ROCA study (NCT-00039559) to assess ROCA's operating characteristics in women at increased familial/genetic risk of ovarian cancer.(28) Subsequently, two ovarian cancer Specialized Program on Research Excellence (SPORE) sites, two Early Detection Research Network (EDRN) sites, and five independent sites opened the study. Together, 25 sites enrolled 2,359 subjects between 2001 and 2011, yielding 6,979 woman-years of screening (median 2.9 yrs; range 0-10.3 yrs). This seemingly short follow-up median duration actually represents 9 screening episodes; 0 years indicates women who enrolled but were never screened. The low median and wide range of duration are due to several CGN sites having additional internal funds enabling screening to continue for longer periods than the other sites. Eligibility criteria included women from families with a deleterious $B R C A 1 / 2$ mutation, and/or multiple ovarian and/or breast cancers in first- or second-degree blood relatives (see Supplement). Women who had previously undergone bilateral oophorectomy $(n=278)$ were eligible for screening for primary peritoneal cancer but were excluded from this analysis. In the CGN study, BRCA1/2 results available at study initiation were recorded but $B R C A 1 / 2$ testing was not performed as part of the study. 
The Gynecologic Oncology Group (GOG) initiated the GOG-0199 study (NCT-00043472) as a twoarm, non-randomized observational study of increased-risk women who chose between risk-reducing salpingo-oophorectomy (RRSO) and ROCA-based ovarian cancer screening. GOG-0199 had the same eligibility criteria as the CGN study, except that women without ovaries were ineligible. The GOG screening arm followed the ROCA protocol, enabling data to be combined. The GOG-0199 screening arm enrolled 1,459 evaluable subjects into the screening cohort across 112 sites in the US and Australia between 2003 and 2006,(27) yielding 6,101 woman-years of screening (median 5.0yrs, range 0-6.9yrs). GOG-0199 participants who were BRCA1/2-unknown at study enrollment underwent research-based germline mutation testing; $B R C A 1 / 2$ mutation status was known to study investigators in $99.6 \%$ of participants. All subjects in both studies signed IRB-approved informed consent.

\section{Screening strategy}

CA125 tests were scheduled every three months. TVU was performed annually regardless of CA125 results as this was considered standard-of-care for high-risk women. The screening strategy implemented $\mathrm{ROCA}(29)$ which individualized the screening test for each woman. For any sequence of CA125 results and test intervals, ROCA calculated the chance (risk) that serum CA125 had a change-point profile which had increased significantly above baseline versus a flat profile which varies stably around the baseline (see Supplement). An increased change-point risk raised suspicion for an undetected tumor. All screening decisions regarding ROCA scheduling or more detailed ultrasound or gynecologic evaluation were based on the ROCA risk level, not the most recent CA125 test result. After each new CA125, ROCA risk was re-calculated, adding the current CA125 to all previous results, subject's age and menopausal status, and the subject was re-triaged: normal-risk 
women ( $<1 \%$ risk of having ovarian cancer) returned in three months for the next CA125; those with an intermediate risk (1-10\%) were referred for TVU; and those with an elevated risk ( $>10 \%)$ received TVU and evaluation by a gynecologic oncologist or study site PI. Consequently, women with abovenormal risks were referred to more intensive follow-up, commensurate with their risk score. The updated ROCA resulted in rapid referral of women with CA125 levels rising significantly above their baseline, including increases within the so-called normal range $(\leq 35 \mathrm{U} / \mathrm{mL})$, to TVU or TVU with gynecologic oncologist review. This strategy avoided further diagnostic evaluation among women with levels $>35 \mathrm{U} / \mathrm{mL}$, but stable compared with their baseline. Thus, the extra information contained in ROCA-interpreted longitudinal CA125 levels potentially increases screening test sensitivity, while retaining the same specificity versus a fixed cut-off applied to the last CA125 value.

All US serum CA125 values were measured by the Massachusetts General Hospital Clinical Laboratory Research Core using the Elecsys CA125-II assay (Roche Diagnostics, Indianapolis, IN).

Since randomized trials with an unscreened arm were judged unethical in women at increased familial/genetic risk, this study compared specificity and PPV with standards set in normal risk populations, compared the proportion of cancers detected in early-stage with published historical results from high-risk women, $(13,14)$ and internally compared ROCA with the single threshold rule of $>35 \mathrm{U} / \mathrm{mL}$ in our data set. Annual specificity of the ROCA blood test was the proportion of women without ovarian cancer not referred to TVU per year. PPV was the proportion of ovarian cancers amongst women undergoing study-indicated pelvic surgery. Early-stage was defined as FIGO (1988) surgical stages $0 / / / I$, since the five-year survival rate in unselected ovarian cancer patients is strongly correlated with stage $(94 \%, 91 \%, 86 \%, 80 \%, 76 \%, 67 \%$, for stages IA, IB, IC, IIA, IIB, IIC, and $45 \%$, $39 \%, 35 \%, 18 \%$ for stages IIIA, IIIB, IIIC, and IV, respectively). The largest survival drop occurs 
between stages IIC and IIIA; most ovarian cancers (66.5\%) are detected as late-stage disease (III/IV). A surgical procedure was considered screen-indicated if it was preceded by an intermediate or elevated ROCA test.

Since women with $B R C A 1 / 2$ mutations are at very high-risk of ovarian cancer, standard care involves strongly considering RRSO following completion of childbearing and at an age when ovarian cancer risk increases above population risk.(5) Study subjects were permitted to elect RRSO in the absence of worrisome symptoms or a positive screening test at any time during the study; 696 subjects in the combined study underwent oophorectomy for any reason while on study. While screening trials for normal-risk women consider the positive predictive value (PPV) - the fraction of ovarian cancers among surgeries following a positive screening test - as the primary efficacy metric, PPV may be less important when a woman at increased risk reaches the point at which standard care recommends RRSO for OC risk management, a practice change which followed two 2005 reports. $(30,31)$ All CGN subjects were followed for $\geq 0 n e$ year after their last screening test with a final questionnaire that ascertained all cancer diagnoses. GOG subjects were planned to undergo 5 years of screening, with cancer outcomes monitored by open-ended annual questionnaires. Invasive ovarian, fallopian tube, or primary peritoneal cancer were the endpoints for this analysis; in aggregate, we designated them "ovarian cancer." Each study had central review of all ovarian surgical specimens(32), including all

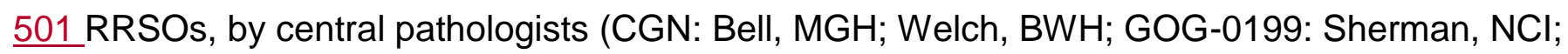
loffe, U Maryland; Ronnett, Johns Hopkins). Among 696 surgical specimens reviewed, there were two instances $(0.3 \%)$ - one from each study - in which an ovarian cancer was identified by central review (GOG: Sherman; CGN: Welch) but not by the study site pathologist. For one of these cases, central review interpreted the lesion as a serous tubal intraepithelial carcinoma (STIC), while the site identified high-grade dysplasia. 


\section{Statistical Methods}

Proportions were compared with standards set from screening normal risk women (specificity, PPV) and historical control reports (sensitivity), using an exact binomial test.(33) Estimates of the proportion of increased-risk women with non-screen-detected early-stage ovarian cancer are difficult to obtain from historical reports. Unlike populations at general risk, there are no registries containing population-based estimates of clinical and pathological features for women at increased risk. Also, there is no standard definition of increased risk, and the understanding of which women are at increased risk has changed over time. Ovarian cancer stage distributions in $B R C A 1 / 2$ germline mutation carriers are a reasonable surrogate for stage distribution in women at increased risk. For comparison with the results in the two screening studies, the population value of the proportion of early-stage invasive ovarian cancers amongst $B R C A 1$ carriers was $10 \%$, calculated from the weighted combination of $8 \%(n=88)$ and $14 \%(n=50)$ from two pathology series reported before screening was common $(13,14)$. Screening studies of women at increased risk(34) differ in their definitions of "increased risk" and/or in their screening regimen, so it is difficult to aggregate their results. We used stage distribution of unselected normal-risk ovarian cancer patients as an additional comparison, ascertained via SEER 92001 tumor registry data, in which the proportion of early-stage disease was 33.5\%, with SEER stages "local" and "regional" corresponding to FIGO stages I \& II.

\section{Results}

\section{Study Population Characteristics}

Table 1 summarizes race and ethnicity, and Table 2 provides ovarian cancer risk factors at baseline. Among 3,449 eligible subjects, $41 \%$ had prior breast cancer, $83 \%$ had a breast cancer family history 
(34\% included one or more premenopausal breast cancers), $47 \%$ had an ovarian cancer family history, and $34 \%$ reported a family history of both. Most subjects were white (92\%), and $20 \%$ were of Ashkenazi Jewish descent. More than half (59\%) were premenopausal, $80 \%$ were parous, $10 \%$ had a pre-enrollment hysterectomy; by self-report, $77 \%$ had used oral contraceptives (median=5 years; range $0-52$ ), and $30 \%$ had used hormone replacement therapy (median=3 years; range $0-44$ ). The CGN cohort comprised $58 \%$ of participants, contributing 6,979 woman-years of screening (53\%), while the GOG cohort contributed 6,101 years of screening. Twenty percent of CGN study participants reported a deleterious mutation. The probability of carrying a $B R C A 1 / 2$ mutation was estimated using $B R C A P R O(35)$ for the CGN cohort, yielding an average of $21 \%$, indicating that reporters of $B R C A$ mutation status were likely representative of the whole CGN cohort. In screening subjects from GOG-0199, 20\% had a documented positive BRCA1/2 test. The similarity in mutation prevalence between the two study cohorts suggests that the common eligibility criteria yielded study groups of equivalent genetic risk. The distribution of variables in Table 1 was similar in both study populations.

\section{Table 1 about here}

\section{Screening Results}

Supplementary Table 1 lists the number of subjects by year for which ROCA was used to evaluate CA125 profiles and triage by risk. On average, $92 \%$ of ROCA evaluations indicated a normal risk (for the study population); these subjects returned for their next regularly scheduled test. Less than $7 \%$ had an intermediate risk and were referred for a study-indicated TVU (92\% annual specificity vs $90 \%$, $\mathrm{p}=0.0001$ ), while $1 \%$ of ROCA tests had an elevated risk level, and were referred to TVU and evaluation by a gynecologic oncologist or study PI (99\% annual specificity). Based on screening model characteristics, we had estimated that $1-2 \%$ of ROCA assays would recommend the highest level of intervention, a rate that was achieved. 
The high frequency of CA125 testing was a crucial screening trial element; we hypothesized that increased frequency would improve detecting early-stage ovarian cancer. The protocol-specified ROCA testing frequency was every three months (i.e., four times/year). The average actual testing frequency (ratio of total CA125 tests to total screening years) was one every four months (three times/year). Each subsequent CA125 test was scheduled three months from last test date if risk was normal, or three months from normal ultrasound date if the risk was intermediate or elevated. Despite the high frequency of testing, $88 \%$ of CA125 tests were conducted within one month of their scheduled time, demonstrating a very high screening compliance rate.

\section{Cancer Outcomes (Sensitivity)}

Table $\underline{2}$ lists the 19 malignant ovarian neoplasms (18 invasive and one intraepithelial carcinoma) identified during the two screening studies: ovary $=8$, fallopian tube $=6$ (including 1 serous tubal intraepithelial carcinoma (STIC)), ovary+fallopian tube $=3$, primary peritoneal carcinoma $=2$. Eleven of 15 serous cancers were high-grade. Three low malignant potential (LMP) tumors, one each in incident, elective RRSO, and prevalent subgroups, were identified, all in stage I; none were known to have a BRCA mutation. The LMPs were omitted from all subsequent analyses. The proportion of study-detected ovarian cancers in early-stage (stages 0/I/I), ROCA detection prior to CA125 >35 $\mathrm{U} / \mathrm{mL}$, and optimal debulking defined the outcomes potentially positively affected by early detection. Screening would not be expected to modify stage at detection of a large proportion of existing but currently undetected tumors, i.e., among prevalent cases (first study-related CA125 test was elevated or part of a rising pattern).(36) Effective screening for incident cases, which arose during rather than before screening initiation, would be expected to yield an increased proportion of early-stage cases. Nine women were diagnosed with ovarian cancers at elective RRSO (none of their screening tests 
had produced a surgical recommendation); thus, we separated surgically-detected cases from screen-detected cases. We analyzed our data stratified by incident, elective RRSO, and prevalent cancer diagnoses. Of 19 ovarian carcinomas identified, six were incident, nine elective RRSO, and four prevalent cases. All prevalent cases were ROCA screen-detected and positive by CA125>35U/mL rule. None of the four prevalent invasive cases was early-stage, statistically commensurate with the historical rate of $10 \%$ early stage disease in high-risk women. Two stage IV prevalent cases were not optimally_debulked. Six (67\%) of the nine RRSO-related carcinoma cases were early-stage, including one non-invasive STIC (Stage 0). All RRSO patients with clinically occult cancers were optimally_debulked.

The six incident cases reflect long-term screening program outcomes; five were screen-detected and one was clinically-detected. Three of the six incident cancers were screen-detected in early-stage (50\%; $95 \% \mathrm{Cl}=12 \%-88 \% ; 50 \%$ versus $10 \%$ historical $B R C A 1$ cases, $p=0.016 ; 50 \%$ versus $33.5 \%$ normal risk cases, $p>0.10$ ). Of the 3 early-stage invasive incident cases, two had a $B R C A 1$ mutation, and one had both $B R C A 1$ and $B R C A 2$ mutations. The two late-stage (IIIC) cases were $B R C A 1 / 2$ mutation-negative. Of the 3 early-stage cases, two were identified when the last CA125 was still $<35$ $\mathrm{U} / \mathrm{mL}$. In 3/6 cases (50\%), ROCA signaled intervention (TVU or TVU plus gynecologic oncologist consultation) prior to CA125>35 U/mL. All 6 incident cases were optimally debulked. Eight of 9 women with stages $0 / / / / l$ ovarian cancers detected in this study were alive at last known follow-up (range 5-9yrs), including the patient with STIC, who is alive and disease-free 5 years after surgery. Of the 3 ROCA-detected stages $\mathrm{I} / \mathrm{II}$ cases, all were alive at follow-up, 2 at 8 years and one at 6 years.

\section{Table 2 about here}

696 subjects had ovarian surgery during the course of study, of whom 195 had surgery preceded by an intermediate or elevated ROCA. Of those surgeries, 9 had ovarian cancer, yielding a conservative 
PPV of $4.6 \%(95 \% \mathrm{Cl}=2.1-8.6 \%)$. Among the 186 false-positive surgeries following a non-normal ROCA, the median age was 48 years. Among the 501 elective RRSOs, there were 9 ovarian cancer cases, yielding an incidence of $2 \%(95 \% \mathrm{Cl}=0.8-3.4 \%)$.

Figure 1 illustrates how longitudinal ROCA testing detected a stage IIB ovarian cancer despite CA125 remaining $<35 \mathrm{U} / \mathrm{mL}$ (red line). ROCA interpreted the CA125 level at the last 2 tests (red circles) as significantly above this woman's baseline, resulting in referral to ultrasound. The second ultrasound was abnormal, which generated a surgical recommendation and diagnosis of a stage IIB ovarian cancer, which was optimally debulked.

\section{Figure 1 about here}

\section{Discussion}

This study shows that ROCA-driven q3 month $\underline{s}$ CA125 testing among increased-risk women was associated with a high specificity $>90 \%$ and a low but possibly acceptable PPV $<10 \%$. This strategy yielded an increased proportion of early-stage invasive ovarian cancer among incident cases compared with historical invasive $B R C A 1$ cases $(50 \%$ versus $10 \% ; p=0.016)$ and compared with cases from the general population ( $50 \%$ versus $33.5 \%$; $p>0.10)$ though not significantly. We have focused sensitivity analysis and discussion on this study sub-group because outcomes for incident cases comprise the best metric for long-term screening. ROCA detected 5/6 (83\%) incident cases, with three (50\%) of the 6 detected prior to CA125 exceeding the standard cut-point of $35 \mathrm{U} / \mathrm{mL}$. These results are commensurate with recent data from the UK Collaborative Trial of Ovarian Cancer Screening (UKCTOCS) general population trial,(36) in which $50 \%$ of incident cases were also detected by ROCA, based on annual CA125 testing prior to CA125>35 U/mL, and $89 \%$ of incident cases were screen-detected.(36) The efficient use of longitudinal CA125 information, with half of the incident cases detected by ROCA prior to CA125 exceeding the standard cut-point, the 3-month 
frequency of scheduled CA125 tests, and using TVU only to triage women with abnormal ROCA results, represent the innovations in the current screening strategy which resulted in a significantly higher proportion of early-stage cases detected in this combined analysis of two screening trials compared with historical $B R C A 1$ controls.

ROCA quantitatively assessed whether recent CA125 results were significantly elevated above each woman's baseline. Figure 1 illustrates a change-point at year 3.5, with a steady increase in five subsequent CA125 tests. In contrast, under the screening rule based on $>35 \mathrm{U} / \mathrm{mL}$ cut-off, no ultrasound would have been performed until after year 6 , by which time the ovarian cancer might have progressed to a more advanced stage. In addition to personalizing the test, our results were obtained using CA125 testing scheduled every 3 months, twice the maximum frequency of CA125 testing that was considered under standard care for increased-risk women.(4) Consequently, 9/10 non-RRSO-related invasive cancers were screen-detected, with only one clinically-detected case (an additional 9 were RRSO-detected), and 17/19 (89\%; 95\%Cl 67\%, 99\%) cancer patients were optimally_debulked; only the two prevalent stage_IV cases were not optimally_debulked. This compares with $58 \%$ optimally_debulked in the unscreened normal-risk population (weighted average from Cochrane Collaboration Report, Table 1: Studies post-2001).(37) Since optimal debulking has been shown to increase survival significantly, $(37,38)$ this may be an additional beneficial outcome for ROCA q3 months screening.

Despite early predictions that women would not comply with testing every three months, a very high proportion (88\%) of CA125 tests was obtained within one month of their scheduled phlebotomy date. On average, our increased-risk study participants underwent CA125 testing every 4 months. These very high compliance rates are encouraging, as they demonstrate the clinical feasibility of this 
intensive strategy, and likely reflect the fact that increased-risk women who choose to retain their ovaries and enroll in a screening trial are very highly motivated to adhere to screening recommendations.

Our study has limitations. Lacking an unscreened control group to which ROCA participants can be compared comprises a significant methodological limitation, but at the time this study was initiated it was judged ethically unacceptable since combined CA125/TVU screening had become the de facto standard of care for increased-risk women, despite unproven efficacy. This represents a difficult methodological constraint: it is unlikely that a prospective, randomized screening trial will be performed in increased-risk women, despite universal recognition that such a design is required to assess disease-specific mortality reduction, the gold-standard screening endpoint. Therefore, stage at detection compared with historical controls was used as a surrogate comparison. However, detecting 3/6 incident carcinoma cases in early-stage does not prove that these women will live longer. UKCTOCS was a randomized screening trial,(25) but it implemented annual ROCA-based CA125 screening in only normal-risk women; its results will only indirectly be related to assessing q3 month $\underline{s}$ ROCA in high-risk women. Our study complements the UKCTOCS report by providing results on increased-risk women. Additional data regarding screen-detected incident cases from ROCA screening of increased-risk populations are needed before our finding of an increase in the proportion of early-stage cases is conclusive. Further studies will also help determine whether interpreting CA125 values with ROCA or the higher q3 months frequency of CA125 testing or both modifications of standard CA125 screening are responsible for the increase in early-stage detection.

A second caveat follows from the low power of our primary analysis, since it is based on only five incident invasive ovarian cancers. But nine additional cancers were detected among women who 
elected RRSO in the absence of symptoms or a ROCA-based recommendation for surgery. Had that option not been available to study participants, the number of analyzable incident events would have been meaningfully larger. We had estimated that $>20$ ovarian cancers would develop in this genetically-predisposed population of women, but the anticipated increased statistical power relative to studying general population subjects was reduced significantly because $14.5 \%(501 / 3,448)$ of participants elected RRSO without a screening-related surgical recommendation, since it is standard practice to counsel genetically at-risk women to consider RRSO once childbearing has been completed and at an age when ovarian cancer risk increases above population risk. Furthermore, when these protocols were designed, $B R C A$-negative women with only breast cancer in their family were study-eligible due to their hypothesized increased risk of ovarian cancer. These women are now not anticipated to be at substantially increased ovarian cancer risk, $(30,39)$ thus further reducing the anticipated power of the study. We are exploring opportunities to pool our results with those of other ROCA-based ovarian cancer screening trials, such as the UK Familial Ovarian Cancer Screening Study (UKFOCSS),(12) in an effort to increase statistical power for sensitivity and PPV.

RRSO cases also present an interpretation issue for screening sensitivity. An alternative interpretation of our data is that the RRSO cases were missed by ROCA, and therefore the sensitivity for early-stage disease for incident cases was 29\% (4/14 = 5 incident ROCA-detected cases +9 cases detected on RRSO) - that is, ROCA missed all 9 RRSO cases (3-25 weeks from last CA125 test to surgery, median 9.3). However, the aim of early detection is to diagnose cancers in early-stage disease, ideally stages 0/I. If the RRSO cases comprised all late-stage disease, then the interpretation that ROCA missed these cases would be reasonable but, in fact, 6/9 RRSO cases were early-stage cancers. RRSO "censored" these cases before they reached late-stage disease. Another analytic alternative would be to combine RRSO cases with incident cases to estimate the proportion 
identified during the screening trial in early-stage disease. This interpretation combines $3 / 6$ incident cases with 6/9 RRSO cases for a combined early-stage proportion of $60 \%(9 / 15 ; 95 \% \mathrm{Cl}: 32 \%-84 \%)$, even higher than the estimate based on incident cases alone (50\%), yields a much tighter confidence interval and therefore greater statistical significance. Thus, we judge our decision to restrict the earlystage estimate to only incident cases as conservative and appropriate.

Another reason we analyzed the RRSO cases separately was because a secondary sensitivity outcome compares ROCA to a CA125 threshold and the RRSO action censored these cases prior to either ROCA being positive or CA125 exceeding $35 \mathrm{U} / \mathrm{mL}$, thus providing no information regarding which occurred first. The large proportion of early-stage cancers among the RRSO cases provides further encouragement to considering RRSO in this population, and reason to hope that some of these women will become long-term, disease-free survivors.

The low $(5 \%)$ screening-related positive predictive value indicates that twenty surgical procedures were performed for each ovarian cancer detected, and comprises a limitation that warrants special comment. We believe this estimate may nonetheless be acceptable among BRCA1/2 mutation carriers after they complete childbearing and reach the age at which RRSO is regarded as standard of care, yet who chose to continue screening. The PPV standard set from consideration of screening trials in the general population is not appropriate in a population for which RRSO is strongly recommended and widely practiced. The low PPV does require caution for women with false-positive results who are below the age at which RRSO is recommended among BRCA1/2 carriers or who have not completed child bearing. Thus, it is reassuring that the median age among false-positive cases was 47 years. 
Due to incomplete $B R C A$ mutation ascertainment in the CGN cohort (a budgetary constraint), our data do not permit drawing conclusions about the utility of this screening strategy in women from mutation-negative/strong family history kindreds. However, among the 19 cancers, there were 13 $B R C A 1,1 B R C A 2$, and 1 subject with a mutation in both genes; 3 were $B R C A 1 / 2$ negative; and 1 subject was untested. Thus, limiting frequent ROCA screening to $B R C A$ mutation carriers would still miss some cases $(3 / 18=17 \%$ of cases tested) in this increased-risk cohort.

Another caveat is that real world application would require adjusting for CA125 variation between laboratories, a concern mitigated by very high inter-laboratory correlation.(40) A further limitation: ROCA screening, even in high-risk populations, requires screening many women to detect a few early-stage cases. In the combined studies, 3 early-stage invasive incident cases were detected that may have been clinically detected in late-stage without screening in 13,080 woman-years, i.e. 23 early-stage cases per 100,000 screened women (0.023\%), a crucial input for a future cost-benefit assessment.

Finally, while ROCA detected cases in an earlier stage than screening with a single CA125 $>35 \mathrm{U} / \mathrm{mL}$ in this study, all were detected in stage II (IIA,IIA,IIB), which has much better survival than stages III/IV, but significantly lower survival than stage I. Better blood tests and secondary imaging must be developed to detect cases in stages 0/I. Many high-grade serous ovarian cancers in BRCA1/2 carriers are believed to originate in the distal fimbriated end of the fallopian tube,(41) and proteins secreted by fallopian tube epithelium may provide promising biomarker candidates.(42) TVU was negative in $3 / 6$ prevalent and $2 / 5$ incident cases, consonant with fallopian tubes being difficult to visualize with TVU and suggesting that a better imaging test is required. The CA125 protein is not shed by $20 \%$ of ovarian cancers, so a CA125-based ROCA cannot detect such cancers. Developing 
a biomarker panel which covers the full ovarian cancer spectrum, and interpreting those data with longitudinal, ROCA-like models, might improve the performance of screening programs aimed at detecting early-stage disease, as shown with FDA-authorized multiple marker diagnostic tests for pelvic masses (ROMA, OVA1).(43, 44) Approaches that may enable earlier detection through analysis of DNA in lower genital tract samples are also under investigation. $(45,46)$

In summary, our study provides the following encouraging evidence: (1) women at increased-risk who agree to an intensive screening regimen are compliant; (2) more frequent CA125 testing interpreted by ROCA is associated with a high specificity and a significant increase in the detection of early-stage incident ovarian cancer compared with published data from historical controls; (3) ROCA detected $\underline{50 \%}$ of incident cases prior to the standard cut-point of $35 \mathrm{U} / \mathrm{mL}$; (4) ROCA detection is associated with a high optimal debulking rate in incident cases; and (5) 8 of 9 women with early-stage cancer were alive at last follow-up. Importantly, we believe these observations do not represent a sufficient basis for introducing this screening strategy into clinical practice as an alternative to RRSO. While even the mixed evidence on the effectiveness of ovarian cancer screening is welcome news, $(36,47)$ we still regard consideration of RRSO upon completion of childbearing and reaching the recommended age as the current standard of care for $B R C A 1 / 2$ mutation carriers. It is essential to recall that even an effective screening program cannot reduce the risk of developing ovarian cancer; its benefit can only derive from earlier detection and improved survival. However, there still remains a significant subset of increased-risk women who choose to retain their ovaries and tubes once their families are complete, despite being fully informed of the benefits of RRSO, including significantly reduced risks of both ovarian cancer and breast cancer, and significantly improved overall survival.(48) Our data suggest that for women who choose screening instead of RRSO, ROCA screening with quarterly CA125 tests, plus TVU as a secondary screen for those with an elevated risk 
score, appeared to be a significant improvement over q6-12 monthly CA125 screening with a single cut-point, such as $35 \mathrm{U} / \mathrm{mL}$. However, due to the small number of incident cases, further evidence from larger cohorts is required before ROCA with q3 months screening tests can be confidently recommended as a replacement for annual or six-monthly testing for women choosing screening. 
Acknowledgements: In addition to the clinical site principal investigators who enrolled more than 50 patients in ROCA and are listed as authors, the following clinical sites and Pl's contributed significant numbers of patients to the study and are gratefully acknowledged: Holly Gallion MD (Magee Women's Hospital), Alex Miller MD (University of Texas Health Sciences Center San Antonio), Paula Ryan MD PhD (Massachusetts General Hospital), Judy Garber MD PhD (Dana Farber Cancer Institute), Henry Lynch MD (Creighton University), James Evans MD PhD (University of North Carolina), Henry Lynch MD (EDRN High Risk Registry), Lee-May Chen MD (University of California San Francisco), Olufunmilayo Olopade MD (University of Chicago), Thomas Caputo MD (Cornell University).

The ROCA study was conducted under the auspices of the Cancer Genetics Network (CGN) with the support of the CGN PI's, which is gratefully acknowledged: Deborah Bowen PhD (Fred Hutchinson Cancer Research Center), Claudine Isaacs MD (Georgetown University), Constance Griffin MD (Johns Hopkins University), Geraldine Mineau PhD (Huntsman Cancer Institute), Joellen Schildkraut PhD (Duke University), Louise Strong MD (MD Anderson Cancer Center), Susan Domchek MD (University of Pennsylvania), Gail Tomlinson MD PhD (University of Texas Southwestern Medical Center), Dennis Ahnen MD (University of Colorado), Hoda Anton-Culver PhD (University of California Irvine), Sharon Plon MD PhD (Baylor College of Medicine), James Evans MD PhD (University of North Carolina), William Wood MD (Emory University), Alex Miller MD (University of Texas Health Sciences Center at San Antonio), Dianne Finkelstein PhD (CGN Coordinating Center, Massachusetts General Hospital), Perry Miller (CGN Medical Informatics, Yale Medical School). In addition, two ovarian SPORE sites and an EDRN site participated with the support of the PI's Edward Partridge (University of Alabama at Birmingham), Robert Ozols (Fox Chase Cancer Center), and Henry Lynch (Creighton University). The Study Coordinators and Research Assistants throughout the CGN, the two ovarian SPORE sites, the EDRN site, and five other sites were crucial to the successful completion of the ROCA study and their tireless efforts are gratefully acknowledged.

The success of GOG-0199 was due to the enormous investment in time and effort made by GOG senior leadership, GOG's Cancer Prevention and Control Committee, the Principal Investigators, Study Managers and Research Assistants from the 150 GOG sites which activated this complex protocol both in the US and Australia, and multiple staff members from the Clinical Genetics Branch, CTEP and CCOP programs at $\mathrm{NCl}$. 
Most importantly, the CGN study and GOG-0199 study would not have been possible without the selfless contributions of the 2,359 (CGN) and 2,605 (GOG) women at increased risk who enrolled in these time-intensive studies. 


\section{References:}

1. Trimble EL. The NIH Consensus Conference on Ovarian Cancer: screening, treatment, and follow-up. Gynecol Oncol. 1994;55:S1-3.

2. Burke W, Daly M, Garber J, Botkin J, Kahn MJ, Lynch P, et al. Recommendations for follow-up care of individuals with an inherited predisposition to cancer. II. BRCA1 and BRCA2. Cancer Genetics Studies Consortium. JAMA : the journal of the American Medical Association. 1997;277:997-1003.

3. Cancer Australia. Surveillance of women at high or potentially high risk of ovarian cancer. 2009 [cited 16 May 2016]; Available from: https://canceraustralia.gov.au/publications-andresources/position-statements/surveillance-women-high-or-potentially-high-risk-ovarian-cancer 4. Daly MB, Axilbund JE, Buys S, Crawford B, Friedman S, Garber JE, et al. NCCN Guidelines Version 1.2012, Genetic/Familial High-Risk Assessment: Breast and Ovarian. NCCN Guidelines. 2012 [cited; Available from: http://www.nccn.org/professionals/physician gls/f guidelines.asp detection

5. Daly MB, Pilarski R, Axilbund JE, Berry M, Buys SS, Crawford B, et al. Genetic/Familial HighRisk Assessment: Breast and Ovarian. NCCN Clinical Practice Guidelines in Oncology (NCCN Guidelines). 2016 [cited; Version 2.2016:[Available from:

https://www.nccn.org/professionals/physician gls/pdf/genetics screening.pdf

6. Evans DG, Gaarenstroom KN, Stirling D, Shenton A, Maehle L, Dorum A, et al. Screening for familial ovarian cancer: poor survival of BRCA1/2 related cancers. J Med Genet. 2009;46:593-7. 7. Woodward ER, Sleightholme HV, Considine AM, Williamson S, McHugo JM, Cruger DG. Annual surveillance by CA125 and transvaginal ultrasound for ovarian cancer in both high-risk and population risk women is ineffective. Bjog. 2007;114:1500-9.

8. Dorum A, Heimdal K, Lovslett K, Kristensen G, Hansen LJ, Sandvei R, et al. Prospectively detected cancer in familial breast/ovarian cancer screening. Acta Obstet Gynecol Scand. 1999;78:906-11.

9. $\quad$ Bourne TH, Campbell S, Reynolds KM, Whitehead MI, Hampson J, Royston P, et al. Screening for early familial ovarian cancer with transvaginal ultrasonography and colour blood flow imaging [see comments]. Bmj. 1993;306:1025-9.

10. Olivier RI, Lubsen-Brandsma MA, Verhoef S, van Beurden M. CA125 and transvaginal ultrasound monitoring in high-risk women cannot prevent the diagnosis of advanced ovarian cancer. Gynecol Oncol. 2006;100:20-6.

11. Hogg R, Friedlander M. Biology of epithelial ovarian cancer: implications for screening women at high genetic risk. J Clin Oncol. 2004;22:1315-27.

12. Rosenthal AN, Fraser L, Manchanda R, Badman P, Philpott S, Mozersky J, et al. Results of annual screening in phase I of the United Kingdom familial ovarian cancer screening study highlight the need for strict adherence to screening schedule. J Clin Oncol. 2013;31:49-57.

13. Rubin SC, Benjamin I, Behbakht K, Takahashi H, Morgan MA, LiVolsi VA, et al. Clinical and pathological features of ovarian cancer in women with germ-line mutations of BRCA1. The New England journal of medicine. 1996;335:1413-6.

14. Boyd J, Sonoda Y, Federici MG, Bogomolniy F, Rhei E, Maresco DL, et al. Clinicopathologic features of BRCA-linked and sporadic ovarian cancer. JAMA. 2000;283:2260-5.

15. Skates SJ, Xu FJ, Yu YH, Sjovall K, Einhorn N, Chang Y, et al. Toward an optimal algorithm for ovarian cancer screening with longitudinal tumor markers. Cancer. 1995;76:2004-10. 
16. Skates SJ, Pauler DK, Jacobs IJ. Screening Based on the Risk of Cancer Calculation from Bayesian Hierarchical Change-Point and Mixture Models of Longitudinal Markers. J Am Stat Assoc. 2001;96:429-39.

17. McIntosh MW, Urban N. A parametric empirical Bayes method for cancer screening using longitudinal observations of a biomarker. Biostatistics. 2003;4:27-40.

18. Skates SJ, Mai P, Horick NK, Piedmonte M, Drescher CW, Isaacs C, et al. Large prospective study of ovarian cancer screening in high-risk women: CA125 cut-point defined by menopausal status. Cancer Prev Res (Phila). 2011;4:1401-8.

19. Daly MB, Axilbund JE, Bryant E, Buys S, Eng C, Friedman S, et al. NCCN Genetic/Familial High-Risk Assessment: Breast and Ovarian. NCCN Clinical Practice Guidelines in Oncology; 2006. p. 1-30.

20. Nezhat FR, Apostol R, Nezhat C, Pejovic T. New insights in the pathophysiology of ovarian cancer and implications for screening and prevention. Am J Obstet Gynecol. 2015;213:262-7.

21. Buys SS, Partridge E, Black A, Johnson CC, Lamerato L, Isaacs C, et al. Effect of screening on ovarian cancer mortality: the Prostate, Lung, Colorectal and Ovarian (PLCO) Cancer Screening Randomized Controlled Trial. JAMA : the journal of the American Medical Association. 2011;305:2295-303.

22. Jacobs IJ, Skates S, Davies AP, Woolas RP, Jeyerajah A, Weidemann P, et al. Risk of diagnosis of ovarian cancer after raised serum CA 125 concentration: a prospective cohort study. Bmj. 1996;313:1355-8.

23. Jacobs IJ, Skates SJ, MacDonald N, Menon U, Rosenthal AN, Davies AP, et al. Screening for ovarian cancer: a pilot randomised controlled trial. Lancet. 1999;353:1207-10.

24. Menon U, Skates SJ, Lewis S, Rosenthal AN, Rufford B, Sibley K, et al. Prospective study using the risk of ovarian cancer algorithm to screen for ovarian cancer. J Clin Oncol. 2005;23:791926.

25. Menon U, Gentry-Maharaj A, Hallett R, Ryan A, Burnell M, Sharma A, et al. Sensitivity and specificity of multimodal and ultrasound screening for ovarian cancer, and stage distribution of detected cancers: results of the prevalence screen of the UK Collaborative Trial of Ovarian Cancer Screening (UKCTOCS). Lancet Oncol. 2009;10:327-40.

26. Menon U, Talaat A, Rosenthal AN, Macdonald ND, Jeyerajah AR, Skates SJ, et al.

Performance of ultrasound as a second line test to serum CA125 in ovarian cancer screening. BJOG. 2014;121 Suppl 7:35-9.

27. Greene MH, Piedmonte M, Alberts D, Gail M, Hensley M, Miner Z, et al. A prospective study of risk-reducing salpingo-oophorectomy and longitudinal CA-125 screening among women at increased genetic risk of ovarian cancer: design and baseline characteristics: a Gynecologic Oncology Group study. Cancer Epidemiol Biomarkers Prev. 2008;17:594-604.

28. Clinical Trial to Screen Participants Who Are at High Genetic Risk for Ovarian Cancer NCT00039559. 2001 [cited; Available from: http://www.ClinicalTrials.gov/ct2/show/NCT00039559

29. Skates S, Pauler D, Jacobs I. Screening Based on the Risk of Cancer Calculation from Bayesian Hierarchical Change-Point and Mixture Models of Longitudinal Markers. J Am Stat Assoc. 2001;96:429-39.

30. Kauff ND, Mitra N, Robson ME, Hurley KE, Chuai S, Goldfrank D, et al. Risk of ovarian cancer in BRCA1 and BRCA2 mutation-negative hereditary breast cancer families. J Natl Cancer Inst. 2005;97:1382-4.

31. Domchek SM, Stopfer JE, Rebbeck TR. Bilateral risk-reducing oophorectomy in BRCA1 and BRCA2 mutation carriers. J Natl Compr Canc Netw. 2006;4:177-82.

32. Sherman ME, Piedmonte M, Mai PL, loffe OB, Ronnett BM, Van Le L, et al. Pathologic findings at risk-reducing salpingo-oophorectomy: primary results from Gynecologic Oncology Group Trial 
GOG-0199. Journal of clinical oncology : official journal of the American Society of Clinical Oncology. 2014;32:3275-83.

33. R Core Team (2015). R: A language and environment for statistical computing. 2015 [cited 2015; Available from: http://www.R-project.org

34. Grover S, Quinn MA, Weideman P, Koh H, Robinson HP, Rome R, et al. Screening for ovarian cancer using serum CA125 and vaginal examination: report on 2550 females. International journal of gynecological cancer : official journal of the International Gynecological Cancer Society. 1995;5:2915.

35. Berry DA, Parmigiani G, Sanchez J, Schildkraut J, Winer E. Probability of carrying a mutation of breast-ovarian cancer gene BRCA1 based on family history [see comments]. J Natl Cancer Inst. 1997;89:227-38.

36. Menon U, Ryan A, Kalsi J, Gentry-Maharaj A, Dawnay A, Habib M, et al. Risk Algorithm Using Serial Biomarker Measurements Doubles the Number of Screen-Detected Cancers Compared With a Single-Threshold Rule in the United Kingdom Collaborative Trial of Ovarian Cancer Screening. J Clin Oncol. 2015;33:2062-71.

37. Elattar A, Bryant A, Winter-Roach BA, Hatem M, Naik R. Optimal primary surgical treatment for advanced epithelial ovarian cancer. The Cochrane database of systematic reviews. 2011:CD007565. 38. Bristow RE, Tomacruz RS, Armstrong DK, Trimble EL, Montz FJ. Survival effect of maximal cytoreductive surgery for advanced ovarian carcinoma during the platinum era: a meta-analysis. Journal of clinical oncology : official journal of the American Society of Clinical Oncology. 2002;20:1248-59.

39. Ingham SL, Warwick J, Buchan I, Sahin S, O'Hara C, Moran A, et al. Ovarian cancer among 8,005 women from a breast cancer family history clinic: no increased risk of invasive ovarian cancer in families testing negative for BRCA1 and BRCA2. Journal of medical genetics. 2013;50:368-72. 40. Mongia SK, Rawlins ML, Owen WE, Roberts WL. Performance characteristics of seven automated CA 125 assays. Am J Clin Pathol. 2006;125:921-7.

41. Lee Y, Miron A, Drapkin R, Nucci MR, Medeiros F, Saleemuddin A, et al. A candidate precursor to serous carcinoma that originates in the distal fallopian tube. The Journal of pathology. 2007;211:26-35.

42. Levanon K, Crum C, Drapkin R. New insights into the pathogenesis of serous ovarian cancer and its clinical impact. Journal of clinical oncology : official journal of the American Society of Clinical Oncology. 2008;26:5284-93.

43. Moore RG, McMeekin DS, Brown AK, DiSilvestro P, Miller MC, Allard WJ, et al. A novel multiple marker bioassay utilizing HE4 and CA125 for the prediction of ovarian cancer in patients with a pelvic mass. Gynecol Oncol. 2009;112:40-6.

44. Zhang Z, Chan DW. The road from discovery to clinical diagnostics: lessons learned from the first FDA-cleared in vitro diagnostic multivariate index assay of proteomic biomarkers. Cancer Epidemiol Biomarkers Prev. 2010;19:2995-9.

45. Kinde I, Bettegowda C, Wang Y, Wu J, Agrawal N, Shih le M, et al. Evaluation of DNA from the Papanicolaou test to detect ovarian and endometrial cancers. Sci Transl Med. 2013;5:167ra4.

46. Erickson BK, Kinde I, Dobbin ZC, Wang Y, Martin JY, Alvarez RD, et al. Detection of somatic TP53 mutations in tampons of patients with high-grade serous ovarian cancer. Obstetrics and gynecology. 2014;124:881-5.

47. Jacobs IJ, Menon U, Ryan A, Gentry-Maharaj A, Burnell M, Kalsi JK, et al. Ovarian cancer screening and mortality in the UK Collaborative Trial of Ovarian Cancer Screening (UKCTOCS): a randomised controlled trial. Lancet. 2016;387:945-56. 
48. Domchek SM, Friebel TM, Singer CF, Evans DG, Lynch HT, Isaacs C, et al. Association of risk-reducing surgery in BRCA1 or BRCA2 mutation carriers with cancer risk and mortality. JAMA : the journal of the American Medical Association. 2010;304:967-75.

Table 1: Race, ethnicity, and ovarian cancer risk factors for all subjects in the two studies

\begin{tabular}{|c|c|c|c|c|}
\hline Variable & $\begin{array}{c}\mathrm{N} \\
(\mathrm{CGN})\end{array}$ & $\begin{array}{c}\text { \% of Total } \\
\text { (CGN) }\end{array}$ & $\begin{array}{c}\mathrm{N} \\
(\mathrm{GOG})\end{array}$ & $\begin{array}{c}\text { \% of Total } \\
\text { (GOG) }\end{array}$ \\
\hline \multicolumn{5}{|l|}{ Race $^{*}$} \\
\hline Asian & 17 & 1 & 16 & 1 \\
\hline Black & 73 & 4 & 33 & 2 \\
\hline White & 1,761 & 88 & 1,399 & 96 \\
\hline Other & 120 & 6 & 10 & 1 \\
\hline Unknown/not reported & 21 & 1 & 0 & 0 \\
\hline \multicolumn{5}{|l|}{ Hispanic Ethnicity } \\
\hline Not Hispanic or Latino & 1,945 & 98 & 1,380 & 95 \\
\hline Hispanic or Latino & 46 & 2 & 21 & 1 \\
\hline Unknown/not reported & 57 & 4 & 0 & 0 \\
\hline \multicolumn{5}{|l|}{ Ashkenazi Jewish Descent } \\
\hline Yes & 365 & 18 & 313 & 21 \\
\hline No & 1,604 & 81 & 1,058 & 73 \\
\hline Unknown & 22 & 1 & 87 & 6 \\
\hline \multicolumn{5}{|l|}{ Menopause Status } \\
\hline Pre-menopause & 1,117 & 56 & 919 & 63 \\
\hline Post-menopause & 874 & 44 & 539 & 37 \\
\hline \multicolumn{5}{|l|}{ Number of Intact Ovaries } \\
\hline 2 & 1,862 & 94 & 1,418 & 97 \\
\hline 1 & 102 & 5 & 40 & 3 \\
\hline Unknown/not reported & 27 & 1 & & \\
\hline \multicolumn{5}{|l|}{ Hysterectomy } \\
\hline Yes & 246 & 12 & 84 & 6 \\
\hline No & 1,715 & 86 & 1,368 & 94 \\
\hline
\end{tabular}




\begin{tabular}{|c|c|c|c|c|}
\hline Unknown & 30 & 2 & 6 & 0 \\
\hline \multicolumn{5}{|l|}{ Ever Pregnant** } \\
\hline Yes & 1,610 & 81 & 1,142 & 78 \\
\hline No & 340 & 17 & 273 & 19 \\
\hline Unknown & 41 & 2 & 43 & 3 \\
\hline \multicolumn{5}{|l|}{ Ever Use Oral Contraceptives } \\
\hline Yes, currently using & 200 & 10 & 176 & 12 \\
\hline Yes, not currently using & 1,354 & 68 & 929 & 64 \\
\hline Never used & 395 & 20 & 353 & 24 \\
\hline Unknown & 42 & 2 & 0 & 0 \\
\hline \multicolumn{5}{|l|}{ Ever Use Hormone Replacement** } \\
\hline Yes, currently using & 202 & 10 & 90 & 6 \\
\hline Yes, not currently using & 442 & 22 & 317 & 22 \\
\hline No & 1,214 & 61 & 1,045 & 72 \\
\hline Unknown & 133 & 7 & 6 & 0 \\
\hline \multicolumn{5}{|l|}{ Personal History of Breast Cancer } \\
\hline Yes & 843 & 42 & 586 & 40 \\
\hline No & 1,122 & 56 & 872 & 60 \\
\hline Unknown & 26 & 1 & 0 & 0 \\
\hline \multicolumn{5}{|l|}{ Family History of Breast Cancer } \\
\hline Yes & 1,669 & 84 & 1,190 & 82 \\
\hline No & 297 & 15 & 241 & 17 \\
\hline Unknown & 25 & 1 & 27 & 2 \\
\hline \multicolumn{5}{|l|}{ Family History of Ovarian Cancer } \\
\hline Yes & 876 & 44 & 745 & 51 \\
\hline No & 1,311 & 66 & 918 & 63 \\
\hline Unknown & 25 & 1 & 16 & 1 \\
\hline \multicolumn{5}{|l|}{ Cohort } \\
\hline & 1,991 & 100 & 1,458 & 100 \\
\hline
\end{tabular}

* With at least one ovary intact at enrollment.

** Percentages may not add to 100 due to rounding. 
Table 2: Ovarian cancers detected during the course of two screening studies

\begin{tabular}{|c|c|c|c|c|c|c|c|c|c|c|c|}
\hline $\begin{array}{c}\text { Case } \\
\# \\
\end{array}$ & $\begin{array}{l}\text { Prevalent } \\
\text { /Incident }\end{array}$ & $\begin{array}{l}\text { Age } \\
\text { at Dx }\end{array}$ & $\begin{array}{l}\text { Mutation } \\
\text { status }\end{array}$ & $\begin{array}{l}\text { Primary } \\
\text { site }\end{array}$ & $\begin{array}{l}\text { ROCA } \\
+ \text { ve }\end{array}$ & $\begin{array}{c}\text { ROCA } \\
\text { vs } \\
>35\end{array}$ & TVU & $\begin{array}{c}\text { Opt } \\
\text { Debulk }\end{array}$ & Histology & Stg & Grd $^{*}$ \\
\hline 1 & Incident & 41 & $\begin{array}{l}B R C A 1+ \\
B R C A 2+\end{array}$ & $\begin{array}{c}\text { Ovary \& } \\
\text { Fallopian tube }\end{array}$ & Y & B & Pos & Y & $\begin{array}{c}\text { Endometrioid } \\
(90 \%), \text { serous, clear } \\
\text { cell }\end{array}$ & IIB & $2-3$ \\
\hline 2 & Incident & 62 & $B R C A 1$ & $\begin{array}{c}\text { Ovary \& } \\
\text { Fallopian tube }\end{array}$ & Y & $=$ & Pos & Y & $\begin{array}{l}\text { Papillary serous } \\
\text { carcinoma }\end{array}$ & IIA & 3 \\
\hline 3 & Incident & 65 & negative & Ovary & $\mathrm{N}$ & NA & Neg & $\mathrm{Y}$ & Serous carcinoma & IIIC & 3 \\
\hline 4 & Incident & 42 & $B R C A 1$ & Fallopian tube & Y & B & NA & Y & $\begin{array}{l}\text { Undifferentiated } \\
\text { carcinoma }\end{array}$ & IIA & 3 \\
\hline 5 & Incident & 64 & negative & Ovary & $\bar{Y}$ & $\mathrm{~B}$ & Pos & $\mathrm{Y}$ & Serous carcinoma & IIIC & 3 \\
\hline 6 & Incident & 49 & $B R C A 1$ & Ovary & $\mathrm{Y}$ & $=$ & $\mathrm{Neg}$ & $\mathrm{Y}$ & Serous carcinoma & $\mathrm{IIIC}$ & 3 \\
\hline 7 & $\begin{array}{c}\text { Elective } \\
\text { RRSO }\end{array}$ & 65 & not tested & Ovary & $\mathrm{N}$ & $\mathrm{N} / \mathrm{A}$ & NA & $\mathrm{Y}$ & $\begin{array}{c}\text { Serous } \\
\text { psammocarcinoma }\end{array}$ & IIIA & 2 \\
\hline 8 & $\begin{array}{l}\text { Elective } \\
\text { RRSO }\end{array}$ & 41 & $B R C A 1$ & Fallopian tube & $\mathrm{N}$ & $\mathrm{N} / \mathrm{A}$ & $\mathrm{Neg}$ & $\begin{array}{c}\text { Not } \\
\text { applicable }\end{array}$ & $\begin{array}{c}\text { Tubal intraepithelial } \\
\text { carcinoma }\end{array}$ & 0 & 0 \\
\hline 9 & $\begin{array}{c}\text { Elective } \\
\text { RRSO }\end{array}$ & 63 & $B R C A 2$ & Ovary & $\mathrm{N}$ & $\mathrm{N} / \mathrm{A}$ & NA & NA & $\begin{array}{l}\text { Endometrioid } \\
\text { carcinoma }\end{array}$ & 1 & 2 \\
\hline 10 & $\begin{array}{l}\text { Elective } \\
\text { RRSO }\end{array}$ & 43 & BRCA1 & Fallopian tube & $\mathrm{N}$ & $\mathrm{N} / \mathrm{A}$ & Neg & Y & Serous carcinoma & IA & 3 \\
\hline 11 & $\begin{array}{c}\text { Elective } \\
\text { RRSO } \\
\end{array}$ & 50 & $B R C A 1$ & Fallopian tube & $\mathrm{N}$ & $\mathrm{N} / \mathrm{A}$ & NA & Y & $\begin{array}{l}\text { carcinoma, } \\
\text { unspecified }\end{array}$ & IC & 3 \\
\hline 12 & $\begin{array}{l}\text { Elective } \\
\text { RRSO } \\
\end{array}$ & 49 & $B R C A 1$ & Ovary & $\mathrm{N}$ & $\mathrm{N} / \mathrm{A}$ & $\mathrm{Neg}$ & Y & Serous carcinoma & IC & 3 \\
\hline 13 & $\begin{array}{l}\text { Elective } \\
\text { RRSO }\end{array}$ & 46 & $B R C A 1$ & Ovary & $\mathrm{N}$ & $\mathrm{N} / \mathrm{A}$ & $\mathrm{Neg}$ & Y & Serous carcinoma & IIIC & 2 \\
\hline 14 & $\begin{array}{l}\text { Elective } \\
\text { RRSO }\end{array}$ & 59 & negative & Fallopian tube & $\mathrm{N}$ & $\mathrm{N} / \mathrm{A}$ & NA & Y & Serous carcinoma & IA & 2 \\
\hline 15 & $\begin{array}{l}\text { Elective } \\
\text { RRSO }\end{array}$ & 42 & $B R C A 1$ & Peritoneum & $\mathrm{N}$ & NA & Neg & Y & Serous carcinoma & III & 3 \\
\hline 16 & Prevalent & 51 & $B R C A 1$ & $\begin{array}{c}\text { Ovary \& } \\
\text { Fallopian tube }\end{array}$ & $\mathrm{Y}$ & $\mathrm{B}$ & $\mathrm{Neg}$ & $\mathrm{N}$ & $\begin{array}{c}\text { Papillary serous } \\
\text { carcinoma }\end{array}$ & IVB & 3 \\
\hline 17 & Prevalent & 40 & $B R C A 1$ & Peritoneum & Y & $=$ & Pos & $\mathrm{N}$ & $\begin{array}{l}\text { Peritoneal serous } \\
\text { carcinoma }\end{array}$ & IIIC & 3 \\
\hline 18 & Prevalent & 48 & $B R C A 1$ & Ovary & $\mathrm{Y}$ & $=$ & Pos & $\mathrm{Y}$ & Serous carcinoma & IIIC & 2 \\
\hline 19 & Prevalent & 81 & $B R C A 1$ & Fallopian tube & $\mathrm{Y}$ & A & Pos & $\mathrm{Y}$ & Serous carcinoma & IIIC & 3 \\
\hline
\end{tabular}

Abbreviations:

Dx: diagnosis

ROCA +ve: ROCA recommended surgical evaluation.

ROCA vs >35: was ROCA abnormal prior to CA125 exceeding $35 \mathrm{U} / \mathrm{mL}$ ?

B: ROCA abnormal before CA125 exceeded $35 \mathrm{U} / \mathrm{mL}$

$=$ : ROCA became abnormal and CA125 exceeded $35 \mathrm{U} / \mathrm{mL}$ simultaneously

A: ROCA became abnormal after CA125 exceeded $35 \mathrm{U} / \mathrm{mL}$

TVU: transvaginal ultrasound

Opt Debulk: patient optimally-debulked

Stg: FIGO stage

Grd: histologic grade

Neg: negative

Pos: positive

NA: not available - ovaries not visualized on TVU

RRSO: risk-reducing salpingo-oophorectomy

* WHO classification divides serous carcinoma into two categories (LG, HG), but WHO classification was not available at the time of pathology review. All Grade 3 serous carcinomas would be HG by WHO. 


\section{LEGENDS}

Figure 1: Early detection of ovarian cancer via ROCA even though CA125 remains below $35 \mathrm{U} / \mathrm{mL}$. The consistent increase in CA125 from the nadir (blue arrow) increases the calculated risk of having ovarian cancer with each additional CA125 test until the risk is elevated (red circles) with a recommendation of a trans-vaginal ultrasound. Surgery was recommended at the second ultrasound and a mixed endometrioid and serous ovarian cancer (stage IIB) detected. 